\title{
La représentation en profondeur de Et les chiens se taisaient d'Aimé Césaire : pour une édition génétique en ligne
}

\section{Alex Gil}

\section{(2) OpenEdition}

\section{Journals}

Édition électronique

URL : http://journals.openedition.org/genesis/605

DOI : $10.4000 /$ genesis.605

ISSN : 2268-1590

Éditeur :

Presses universitaires de Paris Sorbonne (PUPS), Société internationale de génétique artistique littéraire et scientifique (SIGALES)

Édition imprimée

Date de publication : 30 octobre 2011

Pagination : $67-76$

ISBN : 978-2-84050-804-5

ISSN : 1167-5101

\section{Référence électronique}

Alex Gil, « La représentation en profondeur de Et les chiens se taisaient d'Aimé Césaire : pour une édition génétique en ligne », Genesis [En ligne], 33 | 2011, mis en ligne le 23 octobre 2013, consulté le 30 avril 2019. URL : http://journals.openedition.org/genesis/605; DOI : 10.4000/genesis.605 


\section{La représentation en profondeur de Et les chiens se taisaient d'Aimé Césaire : pour une édition génétique en ligne}

Alex Gil

$\mathrm{I}$

est désormais difficile de nier le changement fondamental de paradigme que représente l'édition numérique des textes. Du projet Gutenberg1 de 1971 au projet HyperNietzsche actuellement développé au sein de l'ITEM ${ }^{2}$, les éditions numériques ont évolué d'une simple transcription de texte jusqu'à devenir des outils perfectionnés d'analyse, qui ont changé notre façon de comprendre et d'étudier les textes. Tout au long de ce processus, malheureusement, de nombreux corpus importants de la littérature ont été laissés pour compte. Au cours des années quatre-vingt-dix, la généralisation de l'Internet a coïncidé avec une migration en masse des textes papier vers un support numérique. Mais cet effort étant nécessairement lié à un appui institutionnel et financier massif, la plus grande partie de ces efforts de numérisation a porté sur les canons bibliographiques de l'Amérique du Nord et de l'Europe. D'autres champs littéraires, dont l'immense majorité des corpus de la sphère francophone (Afrique, Caraïbe essentiellement), n'ont pas été pris en compte, pour des raisons diverses (institutionnelles, technologiques, juridiques, etc.) En ce début de $\mathrm{XxI}^{\mathrm{e}}$ siècle, au moment où l'attention s'est déplacée de la numérisation à la création d'outils d'analyse qui pourraient être appliqués à l'énorme quantité de données collectées, il est devenu encore plus difficile d'obtenir des fonds pour les projets de numérisation à grande échelle de ces littératures négligées. Une solution provisoire, donc, consiste à créer des éditions numériques d'œuvres clé de ces grands corpus, en laissant de côté pour le moment la numérisation massive des fonds d'archives. L'édition d'une pièce d'Aimé Césaire se propose donc un but démonstratif et exemplaire. Appelons cette solution la micronumérisation.

Parmi les nombreux défis qui sont à relever par les équipes d'édition numérique, dans le cas d'objets éditoriaux complexes, l'un des plus délicats reste de réussir la collecte et de bien choisir ses stratégies de représentation éditoriale. Les textes de Césaire publiés sous le titre Et les chiens se taisaient sont l'un de ces objets éditoriaux complexes. Réunir dans un seul espace en ligne les matériaux disparates (tapuscrits, livres imprimés, enregistrements, film) qui constituent cette archive sera déjà une réussite considérable. Mais nous désirons ne pas nous arrêter là. Si la tâche de l'édition génétique, comme Walter Benjamin le disait de l'histoire matérialiste, consiste à « prendre possession de la mémoire, dans le cillement d'un éclair au moment du danger ${ }^{3} »-$ en l'occurrence une suite d'actes poétiques réalisés par Aimé Césaire et ses collaborateurs ${ }^{4}$ - alors il nous faut une édition qui puisse : 1) isoler ce que Jerome McGann 5 appelle le code linguistique (les mots) et le code bibliographique (l'arrangement, la typographie, le support matériel, etc.) de façon à ne pas déstabiliser leur équilibre délicat ${ }^{6}$; 2) représenter de façon dynamique la trajectoire et l'interrelation entre les éléments des différents états du texte, afin de nous permettre de recréer le long processus génétique de l'archive. L'absence de coordination entre ces deux fonctionnalités a imposé

1. <www.gutenberg.org/wiki/Main_Page>.

2. <www. hypernietzsche.org/doc/presentation/fr/index.html>.

3. Walter Benjamin, « sich einer Erinnerung bemächtigen, wie sie im Augenblick einer Gefahr aufblitzt », dans Über den Begriff der Geschichte, Illuminationen: Ausgewählte Schriften, Frankfurt am Main, Suhrkamp, 1977.

4. Des interventions éditoriales (i.e. J. Jahn, A. Breton, et al.) que nous incluons ici dans le dossier génétique pris dans son acception large.

5. Jerome McGann, The Textual Condition, Princeton, N.J, Princeton University Press, 1991.

6. Il s'agit ici des seuls objets textuels. Au fur et à mesure que l'édition numérique évoluera et que les éditeurs eux-mêmes rempliront la fonction de conservateur, il est permis d'imaginer un fonctionnement plus sophistiqué de l'archive numérique. 
jusqu'à aujourd'hui une séparation entre les outils d'analyse et la représentation des textes en ligne. Pour nous, l'avenir de l'édition génétique suppose la capacité de joindre l'analyse à la représentation. Les ressources technologiques n'ont pas encore permis de concrétiser toutes nos intentions, mais notre but est de faire reculer les limites actuelles.

\section{Une genèse permanente?}

Dans la mesure où la genèse ne laisse qu'une trace matérielle de l'inscription, et où la continuité textuelle est la répétition substantielle du code linguistique, le processus génétique ne peut être lu qu'en terme d'ajouts, suppressions et transpositions d'éléments du corpus. Tout le reste est conjecture. Ces trois types de manipulation existent en amont et en aval de l'édition princeps. Ces trois mouvements ne deviennent clairs qu'après une manœuvre critique de comparaison. Disons que ce travail de comparaison peut être ou intratextuel (résultant de la comparaison d'un texte donné avec lui-même) ou extratextuel (résultant de la comparaison de deux textes côte à côte.) En comparant des textes dans leur habitus matériel, nous comparons les codes linguistiques et bibliographiques. Mais chaque type de manipulation - ajout, suppression, transposition - influe sur les deux codes de manière différente, et une édition numérique qui reprendrait seulement le code linguistique ne nous montrerait pas le tableau d'ensemble.

Chez bon nombre d'écrivains d'avant-garde - Apollinaire et Pound en offrent d'excellents exemples le code bibliographique jouit d'une importance accrue. Chez Césaire, l'alternance de vers et de prose dans $E t$ les chiens se taisaient mérite tout autant d'attention. L'isolement des codes bibliographiques dans les matériaux originaux ne peut que nous aider à représenter ce qui, dans le texte, est vers et ce qui est prose dans la production de leur milieu premier. Si on pouvait imaginer une édition idéale de cet ouvrage de Césaire, elle devrait nous permettre de comparer ces distinctions de manière significative.

Les chercheurs qui abordent l'historique des textes de Césaire s'étonnent encore du fait qu'il existe de multiples versions de ses ouvrages, chacune portant la marque d'un contexte différent. Pourtant c'est là la norme de l'histoire textuelle des grands auteurs depuis des siècles. Et les chiens se taisaient met la barre un peu plus haut, sans doute, mais cette différence est de degré, non de nature. De plus, Et les chiens se taisaient se trouve à cheval entre la poésie (dans la version de l'oratorio lyrique) et le théâtre (dans la version éditée indépendamment depuis 1956), repoussant ainsi les frontières génériques et proposant une complexité qui dépasse la simple différence entre versions. En somme, cet ouvrage semble tout indiqué pour l'édition génétique en ligne 7 , la seule qui puisse héberger et harmoniser toutes ses versions.

Le premier tapuscrit connu de la pièce (achevé en 1943) traite de la révolution de Saint-Domingue, avec Toussaint Louverture dans le rôle du héros ${ }^{8}$. S'ensuit une période de deux ans environ de refonte intense qui entraîne une première transformation en profondeur. Césaire commence son projet sur la révolution à SaintDomingue sous le régime vichyste de l'amiral Robert (1940 à 1943). Le texte, anticolonialiste et antieuropéen, présente plusieurs indications formelles qui trahissent une intention de porter le sujet à la scène, ce qui était manifestement impossible dans la Martinique verrouillée par le régime militaire et collaborationniste. Le tapuscrit que nous avons retrouvé à la bibliothèque municipale de Saint-Dié des Vosges, qui comporte cent sept feuillets de matière et de format variables, témoigne de multiples faux départs. Dans ce fouillis génétique, on constate des variations dans la situation et le lieu de l'action (de Saint-Domingue à la France, notamment). À une époque qu'il a été impossible de cerner avec toute la précision voulue, Césaire a transformé ce texte primitif (historique et scénique) en un " oratorio lyrique » qui a pris place dans son premier recueil de poésie, Les Armes miraculeuses, édité chez Gallimard en 1946. Dans cette première version éditée de «Et les chiens se taisaient »

7. Une édition sur cédérom ne saurait, en aucune manière, permettre d'atteindre les fins que nous nous proposons ici. Dans notre perspective, la technologie du cédérom est déjà caduque.

8. Voir Alex Gil, « Découverte de l'Urtext de Et les chiens se taisaient », Aimé Césaire à l' œuvre, dir. Marc Cheymol et Philippe Ollé-Laprune, Paris, EAC, 2010, p. 145-156. 
le héros devient le Rebelle, personnage anhistorique, atemporel et dépouillé de toutes les caractéristiques spécifiques à Toussaint Louverture. D'ailleurs la transformation du texte, entouré du contexte lyrique des Armes miraculeuses, a si bien fonctionné que les critiques l'ont rarement lu comme une pièce de théâtre, malgré les didascalies et la présence d'autres personnages plus ou moins nettement définis.

Dans un essai à paraître, Pierre Laforgue conclut, à partir d'une analyse de cette transformation générique, que Césaire a refondu son texte entre 1944 et $1945^{9}$. Laforgue constate, comme nous l'avons fait nous-même, que plusieurs fragments publiés individuellement dans la revue Tropiques se trouvent incorporés à cette version des «Chiens ». Au moins un de ces fragments existe aussi sous forme de tapuscrit. Plus étonnante encore est la reprise de l'un des fragments de Tropiques par la revue Lettres françaises à Buenos Aires sous la direction de R. Caillois en 1945. La transition du tapuscrit à la version éditée sous forme d'oratorio lyrique constitue déjà un défi pour le généticien ; mais ce n’est que le point de départ du processus génétique de cet ouvrage complexe.

Une décennie plus tard, le texte est refondu en Allemagne pour la radio, dans une collaboration extraordinairement étroite entre Césaire et Janheinz Jahn ${ }^{10}$. Jahn intervient en tant que traducteur, certes, mais aussi comme coauteur du texte en langue allemande, ce que Ernstpeter Ruhe a révélé dans une monographie publiée en 199011. Ruhe a démontré à quel point Césaire a accepté les interventions de son collaborateur accompagnant la traduction de son texte pour la radio de l'état de Hesse (Francfort), en 1956. L'appareil critique de son livre ne pouvait représenter qu'une partie des documents qu'il a trouvés dans les archives Jahn conservées par la compagne de celui-ci, Mme Ulla Schild. Une édition génétique en ligne, par contre, offrirait la possibilité de reproduire intégralement tous les documents afférents à cette collaboration, lesquels se trouvent actuellement aux départements d'études africaines de l'université Humboldt, à Berlin, où nous les avons consultés en mai 2010. Cette édition, qui pour le moment reste idéale, comporterait aussi la transcription et l'enregistrement du Hörspiel (pièce radiophonique) de la radio allemande.
La même année, Césaire fait éditer à Paris, chez Présence Africaine, un texte fort différent, conçu pour la scène. Nous savons désormais que ce dernier procède d'un processus de réécriture qui ne doit rien, directement au moins, à la collaboration simultanée avec Jahn. Autre contexte : autre texte. C'est la version que l'on connaît depuis 1956 comme la «version théâtrale » de Et les chiens se taisaient. Un certain nombre d'adaptations s'ensuivirent, dont un court-métrage réalisé par Sarah Maldoror en 1978. Le professeur James Arnold (Université de Virginie) en possède le scénario et nous croyons possible de reproduire également le film, numérisé, dans l'édition en ligne.

Parmi les textes de Césaire écrits pour le théâtre, Et les chiens se taisaient est l'orphelin de la critique. Quoique remis sur le métier vingt fois par Césaire, il rencontre une indifférence quasi totale depuis un demi-siècle, et ceci bien qu'à chaque reprise Césaire ait mis en question la distinction conceptuelle entre adaptation et version. Du tapuscrit à l'oratorio, par exemple, on ne constate pas moins de soixante-quatre transpositions (voir fig. 1). Aux faux départs et tentatives de restructuration notés ci-dessus s'ajoutent de multiples projets de pagination dont on trouve les traces en marge des feuilles. Il importe de noter ici que ces ajouts, suppressions et transpositions ne s'arrêtent pas après la première publication en 1946. Par rapport au texte de l'oratorio lyrique édité dans Les Armes miraculeuses, aussi bien la version allemande à laquelle Jahn a largement contribué que la version théâtrale publiée à Paris en 1956 font preuve d'une quantité impressionnante d'ajouts et de modifications de toute sorte.

\footnotetext{
9. Pierre Laforgue, « Le Cahier d'un retour au pays natal de 1939 à $1947 »$ (texte inédit).

10. À lui seul, Jahn a imposé au cours des années soixante, en Europe et dans les Amériques, la notion d'une civilisation qu'il appelait « néoafricaine », pour désigner les peuples de la diaspora, et dont il a cru pouvoir identifier les constantes, partout dans le monde. On consultera utilement à ce sujet Muntu : l' homme africain et la culture néoafricaine (1961) et son Manuel de littérature néoafricaine du XVIe à nos jours (1969).

11. Ernstpeter Ruhe, Aimé Césaire et Janheinz Jahn, les débuts du théâtre césairien : la nouvelle version de «Et les chiens se taisaient », Würzburg, Königshausen \& Neumann, 1990.
} 


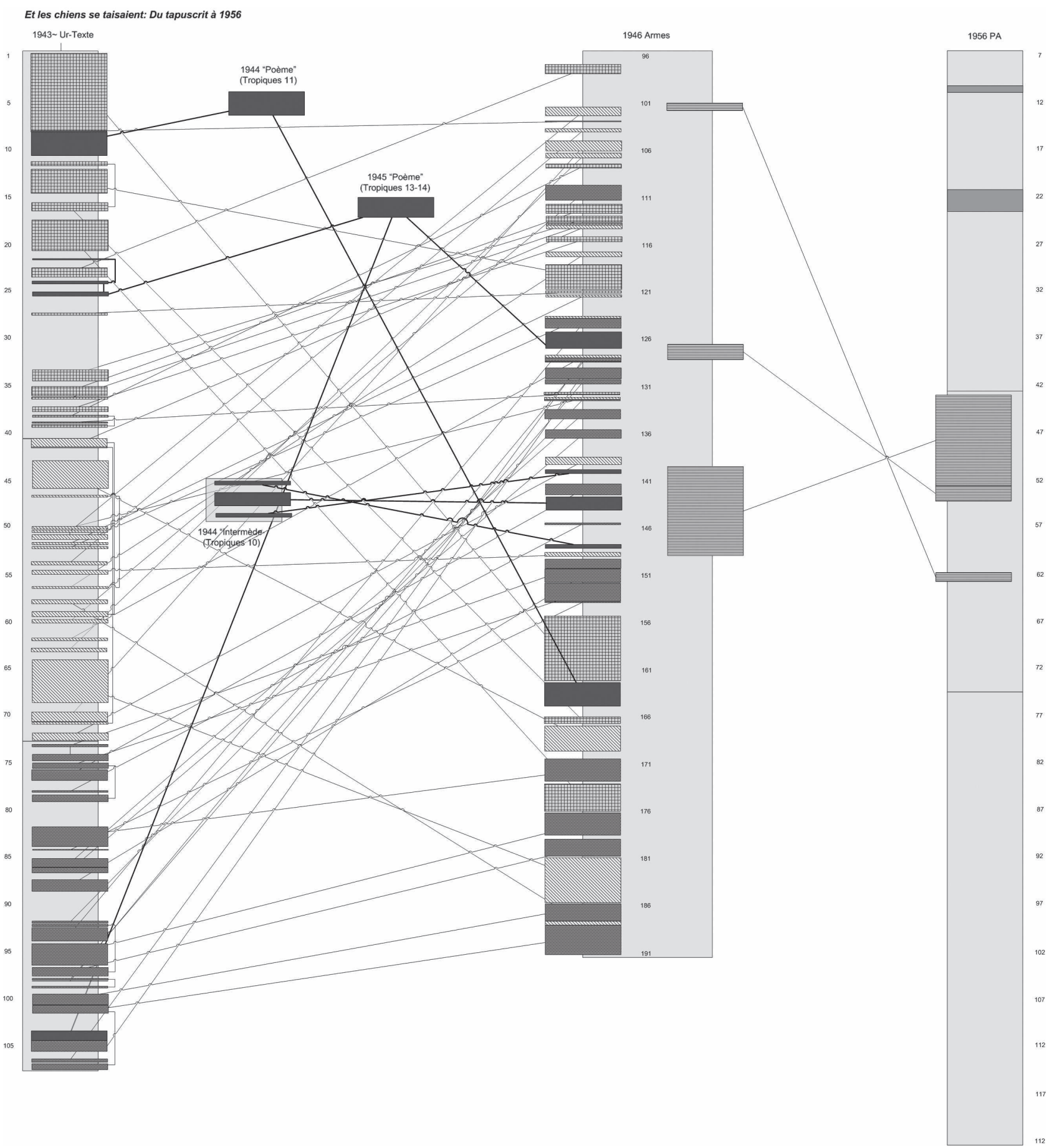

Fig. 1 : Et les chiens se taisaient $:$ du tapuscrit à 1956 
Dans ces conditions, est-il encore possible de faire comme si le processus de création s'arrêtait à la première édition du texte ? Les acrobaties textuelles de Césaire, de 1946 à 1956 autour d'un «même » texte, justifient une analyse de ce que nous entendons par genèse. Si les marqueurs génétiques consistent essentiellement en ajouts, suppressions et transpositions, arrêter l'analyse de ce processus au moment de la première édition nous semble relever d'une distinction quantitative qui dépend du degré d'intensité des interventions auctoriales, non de leur nature. Pourquoi supposer en effet que cette première édition fournirait la stabilité essentielle et posséderait une sorte de valeur iconique qui, par contrecoup, validerait la téléologie des documents de travail ?

Notre analyse des versions successives des Chiens nous mène à croire que Césaire, comme beaucoup d'écrivains avant lui, a montré à ses proches et à ses intimes des versions de son texte bien avant de le faire circuler parmi le public anonyme. Le texte intitulé «Intermède » pourrait nous servir d'exemple. Ce texte a été envoyé par Césaire à Breton, qui à son tour l'a partagé avec son cercle sous forme manuscrite, avant qu'il ne soit publié, d'abord comme un poème individuel, puis dans l'ensemble de l'oratorio lyrique. La quantité des documents de travail antérieurs à la première édition ne doit pas servir d'argument déterminant non plus, car la collaboration de Césaire avec Jahn, comme nous l'avons vu, présente un nouveau « moment » de création bien après la première édition. Sans doute les quelque quatre mille trois cents feuillets manuscrits de Madame Bovary constituent-ils un objet de travail plus enivrant et stimulant, mais les tapuscrits annotés par Césaire sont parfaitement typiques des habitudes de travail de bien des auteurs du $\mathrm{XX}^{\mathrm{e}}$ siècle. Le cas du premier tapuscrit des «Chiens » (notre Urtext) est éloquent quant à la chronologie de sa composition tant il contient d'indices.

Le manuscrit autographe ou bien le tapuscrit annoté ont une vie fort différente de celle du texte publié, certes, mais tous deux sont sujets à des processus propres au texte, dans sa dimension sociale. Une édition génétique en ligne, telle que nous l'envisageons, peut les abstraire pour les comparer. Ainsi, quand Césaire commence à écrire le texte du tapuscrit des « Chiens », son destinataire est évidemment différent de celui visé par le texte publié dans Les Armes miraculeuses. Le public visé par le texte changera à nouveau, au moins deux fois, au cours de l'année 1956, comme nous le démontrerons. Cependant le but reste identique, quoique les moyens varient considérablement. Si notre mission est bien de sauvegarder et restituer les actes poétiques tels qu'ils s'incarnent dans leurs divers habitus, il nous faudra envisager une analyse génétique au long cours.

\section{Blocs de texte : le jeu de lego de Césaire}

L'une des caractéristiques saillantes de la pratique créatrice de Césaire est le réaménagement de ses textes. Il n'est pas le seul à le pratiquer, bien entendu, et tout généticien en reconnaîtra, peu ou prou, les traces chez les auteurs qu'il ou elle édite : en commençant par les balbutiements des débuts pour aller jusqu'aux réimpressions et adaptations posthumes. C'est l'intensité et la fréquence de la démarche qui en fait, en quelque sorte, la signature de Césaire. Au commencement, au moment de la conception - dans la mesure où cette conception s'inscrit et où la trace en reste accessible l'étendue de cette pratique est souvent envahissante. Dans la plupart des cas, nous semble-t-il, cette intensité se détend petit à petit. Dans le cas de Et les chiens se taisaient, nous abordons le texte après le(s) premier(s) brouillon(s), à l'état de tapuscrit, mais l'intensité des transpositions reste toujours très forte, à la fois à l'intérieur du tapuscrit et dans l'édition originale de 1946. Nous appelons bloc textuel le quid de ce processus.

Quoiqu'ils soient très difficiles à cerner et à définir, ces fragments qui creusent et réorientent l'œuvre, à l'intérieur comme à l'extérieur du texte, se laissent pourtant décrire. Ni vraiment mots, ni phrases, ni paragraphes, ce sont des séquences de paroles que les circonstances ont réunies et, pour le meilleur et pour le pire, le sens du texte est lié à leur transposition. On les saisit d'abord à l'aide d'une reconstruction, donc d'un acte critique. À l'intérieur d'un manuscrit autographe ou d'un tapuscrit, on peut les reconnaître par les flèches qui marquent le sens physique de la transposition, mais aussi par l'organisation des pages ou des fragments matériels. 
Dans le cas de Et les chiens se taisaient, nous reconnaissons au moins six paginations différentes qui signalent autant de faux départs ou d'hésitations. À l'intérieur même de ces séquences, nous trouvons des interruptions qui suggèrent que des pages manquent ou ont été retravaillées en profondeur. Trois indices extralinguistiques nous permettent, dans le meilleur des cas, de reconstruire la chronologie des fragments : les instruments (crayon, de couleur ou non, stylo, encre, etc.) utilisés pour effectuer les modifications, le papier (dans ses propriétés matérielles) et la copie au carbone.

L'ordinateur nous permet de reconstruire ces hésitations ainsi que les versions primitives du texte. On pourrait désigner ce processus par le néologisme versionnage virtuel. Une version virtuelle offre bien des possibilités au lecteur comme à l'éditeur. Si le lecteur averti a la capacité de créer au vol, pour ainsi dire, une version du texte qui se conforme aux indices matériels qu'il repère, le processus génétique devient par là même transparent aux yeux du non-spécialiste. Il est évident que le livre imprimé, avec sa logique de continuité qui suppose une finalité, est hostile à ce jeu textuel. Pour revenir au tapuscrit de Et les chiens se taisaient, le versionnage virtuel nous montre neuf étapes de rédaction (voir fig. 2). Nous pouvons observer tout au long de cette reconstruction l'éloignement progressif entre le drame historique originel et le texte surréaliste de l'édition princeps, dès lors que le premier segment a été achevé. Il apparaît donc évident que nous pouvons glaner une quantité considérable d'informations génétiques par cette méthode de figuration du tapuscrit, et que ces informations peuvent s'avérer extrêmement utiles pour envisager des questions plus larges.

La rigueur philosophique qui déstabilise ainsi la présumée autoréférentialité des textes exige sans doute une démonstration concluante. On se convaincra aisément, selon nous, de la différence empirique entre les blocs qui changent de place entre les versions du texte. Cette différence joue à la fois sur le plan interne (bibliographique et linguistique) et sur le plan contextuel, dans la majorité des cas. Il importe d'attirer l'attention sur ce dernier point au moment d'identifier ces entités étranges. À l'évidence, à le considérer dans la perspective du code linguistique d'une seule version, un bloc de texte donné ne se distingue pas de son contexte. Par conséquent, nous devons conclure que le concept de blocs de texte, ainsi définis, existe seulement a posteriori, et résulte de la comparaison de deux versions (virtuelles ou extratextuelles) du texte. La nécessité de juxtaposer des versions du texte entraîne un certain nombre d'observations :

- théoriquement on pourrait appeler bloc de texte tout ce qui dépasse deux vocables mais reste en deçà de la moitié du tout ; toujours sur le plan théorique, au-delà de la moitié, on parle du texte, non de blocs ;

- toutefois, l'analyse de la pratique de transposition révèle rapidement qu'il est impossible d'identifier selon la seule quantification le bloc qui change de position relativement à d'autres parties du texte, car cette démarche génère de nouvelles divisions à l'intérieur du texte conçu comme statique ;

- il est permis dans ces conditions de conclure qu'un bloc de texte est en phase avec son contexte ; ainsi, malgré la corrélation imparfaite entre les fils linguistiques, on peut néanmoins avancer que les blocs correspondent.

Représenter ces transpositions par un moyen qui rende le processus intelligible suppose de pouvoir zoomer sur les deux versions pour les comparer l'une à l'autre. Étant donné la nature linéaire de l'écrit, il faut abstraire les blocs de texte sous forme de figures géométriques pour en faire un graphique didactique. Le but est évidemment de profiter de la mise en place de la littérarité visuelle, sans perte d'informations. Le meilleur exemple actuellement disponible est sans doute celui de Ben Fry. Dans son ouvrage On the Origin of Species: The Preservation of Favoured Traces ${ }^{12}$, Fry révèle comment la représentation par la couleur et les figures géométriques peut permettre la visualisation de modifications textuelles de L'Origine des espèces de Darwin. La faiblesse de sa méthode est de vouloir sauvegarder l'unité idéale du texte. Une approche complémentaire consisterait à juxtaposer les versions. Dans le cas qui nous concerne ici, nous nous trouvons en présence de la transposition du tapuscrit en une version

12. Voir <http://benfry.com/traces/>. 
théâtrale (Présence Africaine, 1956) des Chiens. En utilisant ce même outil de représentation pour l'édition en ligne, nous pourrons combiner le géométrique et le lisible pour mettre en relation les différentes versions du texte.

\section{Représentation profonde et li(vi)sibilité}

Nous appelons représentation profonde de la matérialité des textes dans un milieu cybernétique le type de visualisation que nous venons de décrire. Nous dirons qu'il existe ici deux axes de représentation : la comparaison de deux versions (ou plus) côte à côte correspond à l'axe diachronique (horizontal) parce qu'il représente le mouvement dans le temps. L'axe vertical ou synchronique correspond alors à chaque état individuel du texte, toujours considéré comme le mariage des codes linguistique et bibliographique.

Le développement de l'axe vertical aux fins de publication en ligne présente autant de défis que ceux générés par l'axe horizontal. Dans le cadre du laboratoire de chercheurs Scholars' Lab de l'université de Virginie, nous visons à créer une transformation XSLT qui permettra de coordonner les technologies TEI (p5) et CSS (3.0) afin de générer des transcriptions numérisées qui préserveront, autant que possible, les codes bibliographiques de l'original. Nos expérimentations jusqu'à présent ne nous permettent pas encore de retenir tous les codes bibliographiques d'origine ; la texture du support papier en est un exemple. Dans ces cas, nous continuerons de nous servir de notes basées sur la manipulation de l'original. Les codes que nous arrivons à représenter ainsi comportent des degrés de réussite variables. Parmi les défis auxquels nous devons faire face se trouvent :

- la justification du texte ;

- la présentation de la page ;

- la fonte et la police des caractères ;

- l'espace interlinéaire ;

- la calligraphie ;

- le format matériel de la page (lay-out) ;

- le caractère imparfait (brisé, etc.) du caractère d'imprimerie ou du tapuscrit, et ainsi de suite.
Ces difficultés augmentent, comme on peut l'imaginer, dès qu'on aborde les manuscrits autographes, les tapuscrits et autres brouillons. À court terme, on devra appliquer la politique de transcription des critiques et éditeurs génétiques traditionnels afin de perfectionner la capacité de représentation graphique de l'ordinateur.

Jusqu'à présent, les éditions numérisées ont utilisé des images de l'original (JPEG ou TIFF) afin d'en représenter les codes bibliographiques et n'en ont transcrit que le code linguistique. Les transcriptions ont servi à faciliter la recherche et, dans certains cas, la collation des textes. Alors qu'on possède désormais les applications qui permettent de préciser la cartographie du texte derrière l'image (PDF image/texte par exemple), la question se pose : pourquoi abstraire ? Pourquoi reconstruire les coordonnées textuelles avec HTML ? Ne pourrait-on, au contraire, permettre à l'image de porter le code (TEI) et l'utiliser aux fins de recherche ? La réponse est double : tout d'abord la recherche et la collation ne sont pas les seules opérations dont nous avons besoin. En créant des représentations abstraites comme celles que nous avons illustrées ci-dessus, on pourra créer des versions virtuelles du texte au vol, comme nous l'avons déjà suggéré. Ensuite, une édition génétique bien faite est un instrument didactique qui doit associer l'utile à l'agréable. La transcription du texte par le protocole Unicode, au contraire de la représentation par l'image, réduit la « chaleur» (comme aurait dit le théoricien des médias M. McLuhan) mais nous aide à guider un peu mieux l'attention du lecteur, à manipuler le code linguistique et à créer des versions qui n'ont jamais existé sans un soutien matériel. En fin de compte, l'abstraction réalisée à partir des indices visuels de l'original crée une editio qui produit un supplément d'utilité par rapport à l'original. En plus de la différence inévitable entre l'original et sa reproduction documentaire, on mettra en relief, en les surlignant, des traces qui deviendront ainsi visibles.

C'est à Nicolas Martin-Granel que je suis redevable du merveilleux néologisme li(vi)sibilité, afin de décrire l'heureux entre-deux qui joint le plaisir du texte aux fonctions que le chercheur veut rendre visibles. On ne saurait surestimer l'importance de cette observation. S'il n'est plus nécessaire de sacrifier le plaisir aux opérations scientifiques, il convient de reconnaître que 
la li(vi)sibilité relève de l'interface, non de la quantité d'information en tant que telle. Dans l'état actuel de son développement, l'apparatus criticus est un outil puissant, mais qui exige une maîtrise de toutes sortes de paramètres. La nouvelle édition du logiciel Juxta, à l'origine un logiciel de collation, promet de démultiplier cette fonctionnalité et réduire en même temps la courbe d'apprentissage du chercheur. Ses visualisations communiquent plus rapidement et plus intuitivement que les éditions antérieures. Là où Juxta servait essentiellement d'instrument analytique, sa nouvelle version peut non seulement convertir ses visualisations en images JPEG pour les utiliser n'importe où, mais elle reconnaît désormais aussi l'encodage TEI. Tandis que cette première fonctionnalité nous montre déjà comment Juxta devient un outil de représentation, cette dernière fonctionnalité rendra possible, dans les versions futures du logiciel, de représenter le code bibliographique du texte. La prochaine étape de son évolution portera Juxta directement sur la toile, où les utilisateurs pourront encoder leurs comparaisons directement dans leurs archives, en se passant des images statiques. Pour éviter de donner l'impression de réinventer la roue, une part importante de l'édition de Et les chiens se taisaient portera sur l'interface actuelle de Juxta. Mais avant d'y arriver, il faut mettre en place un certain nombre de structures.

Contrairement à la version courante de Juxta et aux autres applications de type Diff (MEDITE ou la Versioning Machine), qui reconnaissent automatiquement les différences entre les versions de la pièce de Césaire, notre édition inscrira « à la main » l'information codicologique dans l'encodage TEI du texte des Chiens au moyen d'un ancrage non hiérarchique. Afin de communiquer ces codes bibliographiques, ceux-ci doivent être coordonnés ensuite avec le protocole CSS au moyen d'une transformation de type XSLT. Une fois préparés les protocoles CSS et HTML, l'interface fonctionnera sans doute à l'aide de PHP et de JavaScript. Une version future de Juxta rendra ce travail préliminaire obsolète, mais pour le moment ce sont les relations que nous établissons avec le texte de Césaire qui peuvent aider Juxta à se perfectionner. On peut envisager désormais le moment où Juxta - ou un logiciel similaire - deviendra une machine à représenter le texte en profondeur. Le travail en cours est codé de haut en bas en utilisant les normes du standard web, ce qui devra permettre à d'autres projets de se servir directement du modèle. Pour nous, le travail sur les textes de Césaire apporte donc une contribution au monde de l'édition numérique en général.

\section{Deux perspectives : l'interface}

L'interface de Et les chiens se taisaient que nous perfectionnons en ce moment permettra de visionner un seul texte ou deux versions à la fois, comme le fait Juxta actuellement. Dans les deux cas, le produit doit ressembler au texte d'origine. La version simple fournira au lecteur à la fois un texte net et une copie à couches multiples. Le texte net ressemblera à celui que l'on peut télécharger aujourd'hui avec un lecteur numérisé du genre Kindle, iPad ou encore un Smartphone. Si la réalisation est perfectionnée, ce texte comportera une bonne partie de l'information que fournit l'original avec des éléments d'analyse scientifique. La version simple permettra également la création de ces textes qui n'ont pas d'équivalent matériel et que nous appelons virtuels. Pour en donner un exemple, le lecteur pourra produire une version du tapuscrit des Chiens sans les ajouts à l'encre bleue ou sans ajout aucun.

La version à couches multiples admet une complexité accrue. En ajoutant les couches multiples on pourra intégrer des informations appartenant à d'autres versions, afin de créer un texte imaginé dans son unité idéale. De surcroît, les couches multiples seront le support d'annotations scientifiques, de traductions et d'autres types d'appareil critique. La possibilité de travailler avec ces multiples couches d'information permet un type de visualisation du texte poétique qui est autrement plus efficace que le travail sur des textes juxtaposés côte à côte. Au moyen d'un levier chronologique, par exemple, on pourra représenter les ajouts, les suppressions et les transpositions dans un seul et même espace visuel, un peu à la manière du versionnage utilisé par Google Docs aujourd'hui.

Beaucoup des fonctionnalités disponibles dans la version simple peuvent aussi se réaliser dans la version 

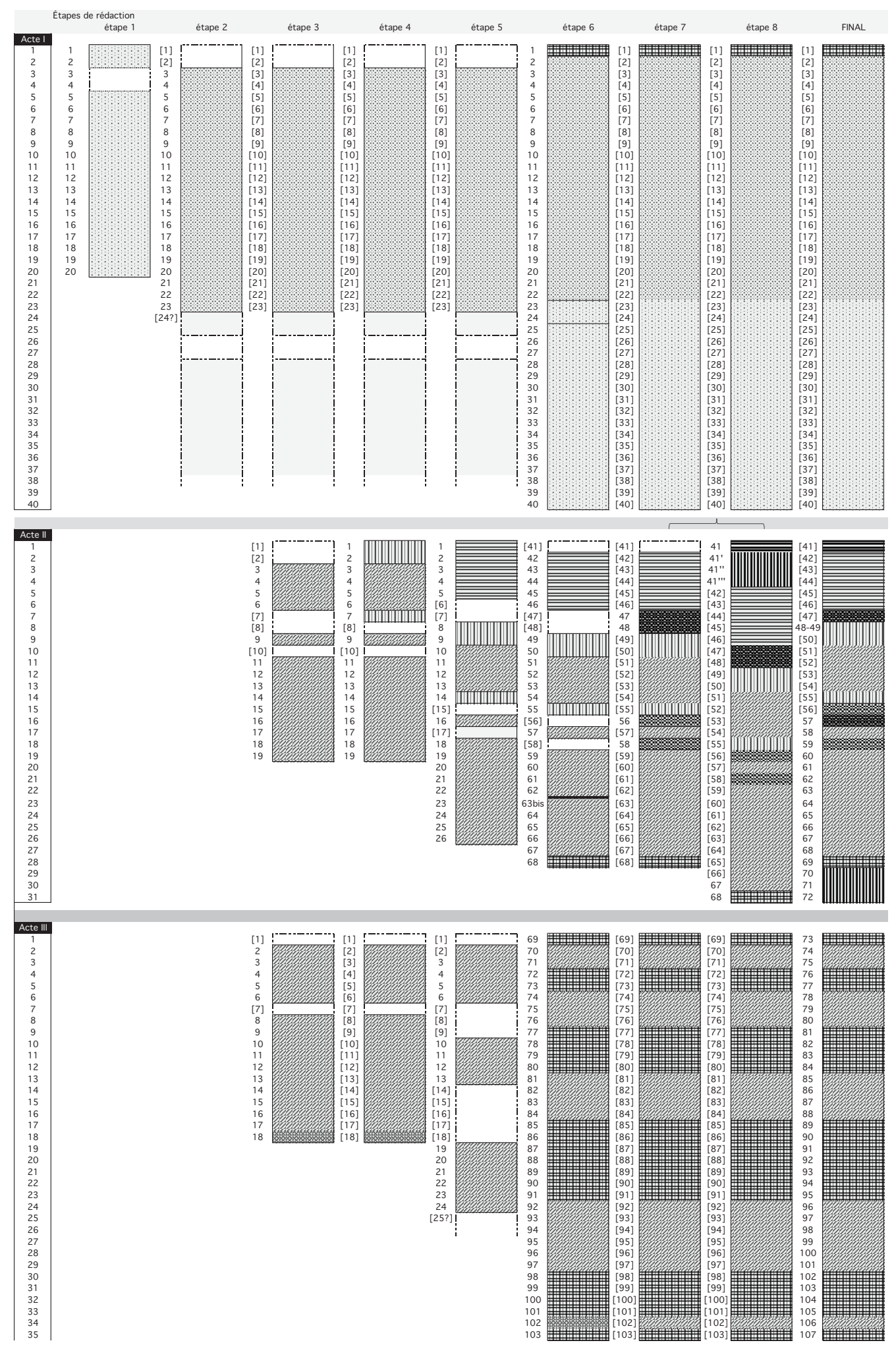

Fig. 2 : Ce tableau représente les différentes configurations du tapuscrit pendant le processus de rédaction. Les trois grands segments horizontaux représentent les actes. Chacune des lignes représente une page du tapuscrit. La première colonne représente la position idéale au sein de l'ensemble.

Chacune des neuf colonnes principales représente une étape différente. La plupart des pages du tapuscrit comportent des traces de numéros de pagination appartenant à des schèmes précédents. Les chiffres figurant à droite de chacune des colonnes principales représentent ces régimes de pagination originale. La dernière étape représente la façon dont nous trouvons le texte dactylographié à Saint-Dié 
double du texte. L'avantage de cette dernière technique de représentation est qu'elle met en relief l'axe diachronique. En minimisant l'emploi des couleurs, cette approche communique aisément les variations dans le temps. L'une des caractéristiques les plus intéressantes de cette représentation de deux textes simultanément est la possibilité de comparer deux versions virtuelles du même tapuscrit ou manuscrit pour nous aider à reconstruire des chronologies de composition. On voit ici comment la logique de l'abstraction est devenue la force motrice du processus. L'action de séparer des éléments discrets du texte en tant que critères analytiques crée une comparaison ceteris paribus. Une autre caractéristique importante de la version double est la possibilité de comparer visuellement les propriétés matérielles de la page ou feuille (format, style, etc.). En créant un codage des propriétés physiques et matérielles de la page ou feuille selon le protocole TEI, on générera des visualisations similaires à celles de Juxta qui isoleront non seulement le code linguistique, mais aussi le code bibliographique. Là encore, le but est de rendre ces caractéristiques utiles autant que possible.

Malgré le degré de complexité que nous apportons à cette interface, le mariage des codes linguistique et bibliographique en est le Saint-Graal. Ainsi l'interface doit s'effacer, aux yeux de l'utilisateur, au profit d'une meilleure représentation du texte. Pour ce faire, on aura besoin des leçons des maîtres du design sur la toile, ce qui est tout autre chose que le design dans l'univers du livre imprimé. Paradoxalement, la solution élégante réalisée dans le domaine de la toile doit servir les besoins de l'univers du livre imprimé. Ce sont finalement les gestes poétiques que nous servons par la création de cette nouvelle technologie. L'interface n'est qu'un instrument sophistiqué certes - qui nous permet d'approfondir notre engagement envers ces gestes poétiques.

Alex GIL présentera prochainement, au département d'anglais de l'université de Virginie (Charlottesville), sa thèse comparatiste sur la littérature de l'aire caraïbe. Dans le cadre de cette thèse, il prépare une édition variorum numérique de Et les chiens se taisaient d'Aimé Césaire. Membre de l'équipe éditoriale du projet Césaire de l'AUF-ITEM, il a terminé plusieurs articles sur les premières œuvres de Césaire.

Alex Gil, colibri.alex@gmail.com 


\section{La représentation en profondeur de Et les chiens se taisaient d'Aimé Césaire : pour une édition génétique en ligne}

Les nouvelles possibilités qu'offre l'édition numérique nous obligent à repenser l'analyse des différents états d'une œuvre avant et après sa première publication. Le texte d'Aimé Césaire intitulé Et les chiens se taisaient nous servira d'exemple. Il ressort de cet examen que l'on ne saurait interrompre l'étude de la genèse à l'édition originale du texte. La pratique rédactionnelle de Césaire est caractérisée par une surabondance de transpositions de blocs de texte d'une version à l'autre. Son œuvre offre par conséquent un champ particulièrement propice à l'étude des blocs de texte en tant que catégorie analytique. L'édition numérisée se prête à cette opération par sa capacité à mettre en évidence les transformations du texte. Nous proposons une technologie spécifique qui permettra de créer une édition complexe combinant analyse et représentation, et de révéler, par l'affichage simultané des différentes versions du texte, le processus interne de création.

The new possibilities that digital editions offer require us to re-examine the analysis of a work's various stages, before and after its first publication. We will use Aimé Césaire's text titled $E t$ les chiens se taisaient as an example. The outcome of this study shows that the genetic study of a text does not stop at its original edition. Césaire's writing practice is defined by an overabundance of text units shifting from one version to another. Consequently, his work provides a particularly favorable field for the study of text units considered as an analytical category. Digital edition lends itself to this process thanks to its capacity to highlight the text's transformations. We suggest a specific technology that will make possible a complex edition combining analysis and representation and, with a simultaneous display of the text's various versions, revealing the inner creative process.

Die neuen Möglichkeiten, die sich bei einer digitalen Ausgabe bieten, nötigen uns, die Analyse der verschiedenen Zustände eines Werkes vor und nach dessen erster Veröffentlichung neu zu überdenken. Der Text von Aimé Césaire mit dem Titel Et les chiens se taisent dient uns als Beispiel. Es zeigt sich bei dessen Untersuchung, dass man bei der genetischen Analyse nicht bei der Edition des Originaltextes stehenbleiben kann. Charakteristisch für die redaktionelle Praxis Césaires ist die Überfülle von Transpositionen von Textblöcken, von einer Version zur nächsten. Sein Werk bietet deshalb ein besonders ergiebiges Feld für die Untersuchung von Textblöcken als analytische Kategorie. Die digitale Edition bietet sich bei diesem Unterfangen an, da sie es ermöglicht, die Texttransformationen darzustellen. Wir schlagen deshalb eine spezielle Technik vor, die es erlauben wird, eine komplexe Ausgabe zu erstellen, die Analyse und Darstellung miteinander verbindet, und durch die gleichzeitige Darstellung verschiedener Textversionen den inneren Schaffensprozess aufzuzeigen.
Las nuevas posibilidades que brinda la edición numérica nos obligan a reconsiderar el análisis de los diferentes estados de una obra antes y después de su primera publicación. El texto de Aimé Césaire titulado Et les chiens se taisaient nos servirá como ejemplo. Este examen pone de manifiesto la necesidad de prolongar el estudio de la génesis más allá de la edición original del texto. La práctica redaccional de Césaire se caracteriza por una superabundancia de transposiciones de bloques de textos de una versión a la otra, por lo cual, su obra ofrece un campo particularmente propicio al estudio de los bloques de texto en tanto que categoría analítica. La edición digital se presta a esta operación, por su capacidad a poner en evidencia las transformaciones del texto. Proponemos una tecnología específica que permitirá crear una edición compleja, combinando análisis y representación, y revelar, a través de la visualización simultánea de diferentes versiones del texto, el proceso interno de creación.

Le nuove possibilità che offre l'edizione digitale ci obbliga a ripensare l'analisi dei diversi momenti di un'opera, prima e dopo la sua prima pubblicazione. Il testo di Aimé Césaire intitolato Et les chiens se taisaient ci servira d'esempio. Da questo esame emerge che non si deve interrompere lo studio genetico quando si arriva all'edizione originale dell'opera. La pratica redazionale di Césaire è caratterizzata da una sovrabbondanza di trasposizioni di blocchi di testo da una versione all'altra. Di conseguenza la sua opera offre un campo particolarmente propizio allo studio dei blocchi di testo come categoria di analisi. L'edizione digitale ben si presta a questa operazione per la sua capacità di mettere in luce le trasformazioni del testo. Qui si propone una tecnologia specifica che permetterà di realizzare un'edizione complessa che combina analisi e rappresentazione, e di rivelare, per mezzo della presentazione simultanea di versioni differenti del testo, il processo interno di creazione.

As novas possibilidades que oferece a edição digital obrigam-nos a reconsiderar a análise dos diferentes estados de uma obra antes e após da sua primeira publicação. O texto de Aimé Césaire intitulado Et les chiens se taisaient servirá de exemplo. Resulta desse exame que não se poderia interromper o estudo da génese na edição original do texto. A prática redaccional de Césaire é caracterizada por abundantes transposições de blocos de texto de uma versão para outra. A sua obra oferece por conseguinte um campo particularmente propício ao estudo dos blocos de texto como categoria analítica. A edição digital presta-se a essa operação pela capacidade de realçar as transformações do texto. Propomos uma tecnologia específica que permitirá criar uma edição complexa que combina análise e representação, e revela, pela afixação simultânea das diferentes versões do texto, o processo interno da criação. 